\title{
Reduction in Ventral Midbrain NMDA Receptors Reveals Two Opposite Modulatory Roles for Glutamate on Reward
}

\author{
Giovanni Hernandez', Ali Khodami-Pour ${ }^{2}$, Daniel Lévesque ${ }^{3}$ and Pierre-Paul Rompré*,1,2 \\ 'Département de Neurosciences, Université de Montréal, Montréal, QC, Canada; ${ }^{2}$ FRQ-S Research Group in Behavioral Neurobiology, \\ Department of Psychology, Concordia University, Montréal, QC, Canada; ${ }^{3}$ Faculté de Pharmacie, Université de Montréal, Montréal, QC, Canada
}

\begin{abstract}
Glutamate is a major component of the reward circuitry and recent clinical studies suggest that new molecules that would target glutamate neurotransmission are most likely to constitute more effective medications for mood disorders. It is well known that activation of N-methyl-D-aspartate glutamate receptors (NMDARs) initiates dopamine burst firing, a mode associated with reward signaling; but NMDARs also contribute to the maintenance of an inhibitory drive to dopamine neurons. Such opposite modulatory functions imply that different subtypes of NMDARs are expressed on different ventral midbrain (VM) neurons and/or afferent inputs to dopamine neurons. By using the small interfering RNA (siRNA) technique, we studied the effects of VM downregulation of NMDAR subunits GluNI, GluN2A, and GluN2D on reward induced by dorsal raphe electrical stimulation. Reward thresholds were measured before and $24 \mathrm{~h}$ after each of three consecutive daily bilateral microinjections of siRNA for the targeted receptor subunit(s) or non-active RNA sequence. After the last measurement, reward thresholds were reassessed following a bilateral microinjection of the preferred GluN2A-NMDA antagonist, (2R,4S)-4-(3-Phosphopropyl)-2-piperidinecarboxylic acid (PPPA). Western-blot analysis showed that siRNAs reduced GluNI - and GluN2A-containing receptors whereas behavioral tests showed that only a reduction in GluNI produced reward attenuation. Despite NMDAR reduction, reward-enhancing effect of PPPA remained unchanged. We conclude that VM glutamate relays the reward signal initiated by dorsal raphe electrical stimulation by acting on NMDARs devoid of GluN2A/2D subunits and exerts an inhibition on this reward signal by acting on GluN2A-containing NMDARs most likely located on afferent terminals.

Neuropsychopharmacology (20I5) 40, I682-169I; doi:I0.1038/npp.20I5.|4; published online II February 2015
\end{abstract}

\section{INTRODUCTION}

Depression is the most common mental illness affecting around 350 million people worldwide. It is expected to become the second most common cause of disability worldwide by 2015 with obvious socio-economic implications (Strekalova et al, 2011). Traditional pharmacotherapy which focuses on increasing monoamines (norepinephrine or serotonin) neurotransmission has a relatively slow onset (Kupfer et al, 1989) of action and it has a limited effectiveness, between 10 and $20 \%$ of the treated patients do not respond to any antidepressant medication (Greenberg et al, 2004). Recent clinical studies have shown that a single subanesthetic dosage of the non-competitive N-methyl-Daspartate receptor (NMDARs) antagonist, ketamine, produces a rapid and lasting antidepressant effect in patients who were resistant to the current treatment (Maeng et al, 2008). These findings suggest that glutamate receptors might

\footnotetext{
* Correspondence: Dr P-P Rompré, Département de Neurosciences, Université de Montréal, C.P. 6128, Succ Centre-Ville, Montréal, QC H3C 3J7, Canada, Tel: + I 5143436111 (extn 3535I), Fax: + 514 343 7972, E-mail: pierre-paul.rompre@umontreal.ca

Received I5 October 2014; revised 16 December 2014; accepted 29 December 2014; accepted article preview online 12 January 2015
}

represent a very promising pharmacological approach to alleviate depressive symptoms.

Anhedonia, a core symptom of depression, is associated with a downregulation of the mesolimbic dopaminergic pathway (Nestler and Carlezon, 2006) and NMDARs antagonists may ameliorate mood disorders through the modulation of ventral midbrain (VM) dopamine (DA) neurons. Under resting condition, about half of DA neurons are under a strong tonic inhibitory drive that makes them hardly responsive to excitatory inputs (Grace and Bunney, 1984). The other half of DA neurons are spontaneously active and fire in two distinctive modes: an intrinsic sustained slow tonic firing or a fast phasic burst firing. The switch from tonic to phasic firing is controlled by glutamate signaling at its ionotropic receptors. Activation of glutamatergic afferents (Geisler et al, 2007; Grace and Bunney, 1984; Omelchenko and Sesack, 2009), and possibly VM VGluT2-containing interneurons (Dobi et al, 2010), control the transition from peacemaker tonic to phasic burst firing of DA neurons. In contrast, blockade of VM ionotropic glutamate receptors disrupts burst firing (Charlety et al, 1991; Chergui et al, 1993). Dopamine burst firing signals the occurrence of salient unconditional and conditional stimuli (Schultz, 2010) and it is believed to convey motivationally relevant information to forebrain structures involved in motor execution 
(Overton and Clark, 1997). In addition DA burst firing is critical for learning of appetitive and aversive task (Zweifel et al, 2009) and it is believed to encode learning prediction errors (Schultz, 2010).

Besides its role in firing mode transition, glutamate also has a role in the maintenance of DA inhibitory drive (Grace and Bunney, 1984) and a reduction of this tonic inhibition allow an increased number of DA neurons to fire in a sustained slow tonic firing and in a fast phasic burst firing. These two opposite modulatory functions of glutamate imply that different subtypes of NMDARs are activated by different inputs to VM DA neurons, so that activation of one subtype of NMDAR initiates DA burst firing whereas activation of another enhances the inhibitory input to DA neurons. This idea is supported by data showing that either activation or blockade of VM NMDARs increases DA burst firing (French et al, 1993), accumbens DA release (Karreman et al, 1996; Kretschmer, 1999), and stimulates forward locomotion (Cornish et al, 2001; Kretschmer, 1999). Blockade of VM NMDARs also acts as a positive reinforcer and sustains self-administration behavior (David et al, 1998). Furthermore, VM microinjections of the PPPA and R-CPP, NMDAR antagonists for GluN2A-D subunits, potentiate the rewarding effect of electrical brain stimulation; such a potentiation was not seen with Ro 04-5595, a selective GluN2B antagonist, hence suggesting that glutamate exerts an inhibitory modulation on VM reward-relevant neurons by acting on NMDARs composed of GluN2A and/or GluN2D (Bergeron and Rompré, 2013). The present study was thus aimed at further characterizing the NMDARs that is/are responsible for this inhibitory modulation of reward. Using the curve-shift method, we studied the effects of VM downregulation of NMDAR subunits GluN1, GluN2A, GluN2D, and GluN2A + D, using a small interfering RNA (siRNA), on reward induced by electrical brain stimulation. Results show that VM glutamate relays the reward signal initiated by the electrical stimulation by acting on receptors devoid of GluN2A/2D subunits and exerts an inhibition on reward signaling by acting on GluN2A NMDARs that are most likely located on VM afferent terminals.

\section{MATERIALS AND METHODS}

\section{Subjects and Surgery}

Fifty (50) male Long-Evans rats (Charles River, St Constant, QC, Canada) weighing between 350 and $400 \mathrm{~g}$ at the time of the surgery were used as experimental subjects. They were individually housed in a temperature and humidity controlled room with a 12-h light-dark cycle (lights on at $06: 00 \mathrm{~h}$ ) with ad-libitum access to food and water. After a minimum 7 days period of acclimation to the housing environment rats were anesthetized with isoflurane (2.5$3.5 \% \mathrm{O}_{2}, 0.61 / \mathrm{min}$ ) and stereotaxically implanted according to Paxinos and Watson (2007) coordinates with 26-gauge guided cannulae (Model C315G HRS Scientific, Montreal, Canada) aimed bilaterally above the $\mathrm{VM}(-5.5 \mathrm{~mm} \mathrm{AP}$, $3.2 \mathrm{~mm} \mathrm{ML}$ at a $18^{\circ}$ angle, $6.5 \mathrm{~mm} \mathrm{DV}$ from the skull surface) and a movable monopolar electrode aimed at the dorsal raphe $(-7.6 \mathrm{~mm} \mathrm{AP,} 0.0 \mathrm{~mm} \mathrm{ML}, 6.6 \mathrm{~mm} \mathrm{DV}$ from the skull surface). See Bergeron and Rompré (2013) for further surgery details. All procedures were carried out in accordance with established practices as described in the NIH Guide for Care and Use of Laboratory Animals. In addition, all procedures were reviewed and approved by the Animal Care and Use Committee of Université de Montréal.

\section{Self-Stimulation Training}

Each of the rats was shaped to nose-poke for a $0.4 \mathrm{~s}$ train of cathodal, rectangular, constant-current pulses, $0.1 \mathrm{~ms}$ in duration, delivered at a frequency of $98 \mathrm{~Hz}$. For a detailed shaping procedure see Bergeron and Rompré (2013). Once the rat nose-poked consistently for currents between 125 and $400 \mu \mathrm{A}$, a rate $v s$ pulse-frequency curve was obtained by varying the stimulation frequency across trials over a range that drove the number of rewards earned from maximal to minimal levels. Each trial for obtaining the rate $v s$ pulsefrequency sweep lasted for $55 \mathrm{~s}$ followed by a 15-s inter-trial interval during which stimulation was not available. The beginning of each trial was signaled by five trains of noncontingent priming stimulation delivered at a rate of 1 per second. Four sweeps were run daily and the first sweep was considered warmup and discarded from analysis. The data relating the rate to the pulse frequency was fitted to a sigmoid and the index of reward threshold defined as the pulse frequency sustaining a half-maximal rate of responding (M50) was obtained (see Supplementary Figure 1 for a diagram of the procedure).

\section{Self-Stimulation Tests}

Prior to the siRNA injections, sterile $0.9 \%$ saline was microinjected in the VM to habituate the animals to the injection procedure. Bilateral injections were made by inserting an injection cannula (Model C315I HRS Scientific, Montreal, Canada) that extended $2 \mathrm{~mm}$ beyond the guide cannula tip. The injection cannula was connected to a $5 \mu \mathrm{l}$ Hamilton micro-syringe via polyethylene tubing. A total volume of $0.5 \mu \mathrm{l}$ was injected in each hemisphere simultaneously over a 60 -s period. The rate of delivery was controlled by an infusion pump (Harvard Instruments, Holliston, MA). To allow diffusion into the tissue the injection cannulae were left in place for additional $60 \mathrm{~s}$. After the microinjection, the subjects were put into the operant boxes and allowed to self-stimulate. Results from this test were not included in the analysis. Baseline data were collected 1 week after this first saline microinjection and the self-stimulation behavior was considered stable when the M50 values varied less than one $0.1 \log$ unit for three consecutive days. Once stable baseline was obtained siRNA (5 $\mu \mathrm{g}$ per side) against GluN1, GluN2A, GluN2D, GluN2A + GluN2D, or the non-active RNA sequence was injected bilaterally into the VM for three consecutive days. Reward thresholds were measured $24 \mathrm{~h}$ after each injection. After $24 \mathrm{~h}$ the last threshold determination rats received a bilateral VM microinjection of the NMDA antagonist PPPA $(0.825 \mathrm{nmol}$ per $0.5 \mu \mathrm{l}$ per side) and reward thresholds were measured again immediately after the injection for $120 \mathrm{~min}$.

\section{siRNA and Drug}

Downregulation of selective NMDAR subunits was achieved using a mixture of pre-validated siRNA sequences against 
rat GluN1 (Cat. \#4390816 ID s127804- s127806), GluN2A (Cat. \#4390816 ID s127807- s127809), and/or GluN2D (Cat. \#4390816 ID s127816- s127818) receptors. A non-active RNA sequence (Cat. \#4390844) (scramble) was used as control. siRNA sequences and non-active RNA sequence were purchased from Ambion (Austin, TX) as control. The deprotected, duplexed, desalted siRNA was mixed with a cationic lipid transfection carrier N-[1-(2,3-Dioleoyloxy)propyl]-N,N,N-trimethyl-ammonium methylsulfate (DOTAP) (Roche Applied Sciences, Indianapolis, IN) which showed high efficacy for in-vivo transfection (Salahpour et al, 2007). The final solution contained $10 \mu \mathrm{g}$ of the active or inactive siRNA and $1 \mu \mathrm{g}$ of DOTAP per $\mu \mathrm{l}$. NMDAR antagonist PPPA ((2R,4S)-4-(3-Phosphopropyl)-2-piperidinecarboxylic acid) was purchased from Tocris Bioscience (Ellisville, MI, USA). The drug was dissolved in sterile $0.9 \%$ saline and stored frozen in $40-50 \mu \mathrm{l}$ aliquots. Drug solutions were thawed just prior to testing and injected at a dose of $0.825 \mathrm{nmol}$ per $0.5 \mu \mathrm{l}$ per side. Dose is expressed as salt.

\section{Western-Blot}

Rats were decapitated immediately after the last behavioral test. Brains were removed and immediately placed on an ice-cold brain matrix, and sectioned coronally. The VM was dissected on an ice-cooled plate from a $0.75-1-\mathrm{mm}$ slice using a 15-gauge tissue punch. The tissue was then immediately frozen at $-80^{\circ} \mathrm{C}$ until biochemical experiments were performed. VM samples were mechanically homogenized in lysis buffer (10 mm Tris-HCl ( $\mathrm{pH} 6.8), 2 \%$ SDS, and a cocktail protease inhibitor (Roche)). The protein concentrations of the tissue samples were measured using BCA protein assay kit (Pierce, USA). Equal amounts of protein $(10-50 \mu \mathrm{g})$ were dissolved into $25 \mu \mathrm{l}$ lysis buffer (which contained $4.5 \mu \mathrm{l} 5 \times$ loading buffer and $0.5 \beta$-mercaptoethanol), boiled at $95^{\circ} \mathrm{C}$ for $5 \mathrm{~min}$, and then subjected to SDS-PAGE with $8 \%$ polyacrylamide gels and transferred to PVDF membrane (Bio-Rad Laboratories). The membranes were blocked for $1 \mathrm{~h}$ in TBST with 5\% dry milk and were incubated with mouse anti- $\beta$-actin (Millipore) at $1: 10000$ with either rabbit anti-GluN1 (Novus Biological) at $1: 500$, anti-GluN2A (Cayman) at 1:1000, anti-Glun2B (Novus Biological) at 1:1000, or anti-GluN2D (Santa Cruz Biotechnology) at $1: 100$ overnight at $4{ }^{\circ} \mathrm{C}$. After rinsing four times with TBST for $5 \mathrm{~min}$, the membranes were incubated in HRP-conjugated goat anti-Rabbit IgG (Millipore) at 1:6000 (to detect GluN1, GluN2A, GluN2B, GluN2D) or HRPconjugated goat anti-Mouse IgG (Millipore) at 1:6000 (to detect $\beta$-actin, GluN2D) for $1 \mathrm{~h}$. The membrane protein bands were detected with ECL western blotting system (PerkinElmer) and visualized on an X-ray film. The band densities were measured with Image $(\mathrm{NIH})$ and were normalized to $\beta$-actin. The protein levels of the different subunits of the NMDAR were expressed as a percentage of control.

\section{Data Analysis}

Levels of protein were contrasted using Student's $t$-test. Fit of the rate-frequency data and M50 value were obtained using Matlab (Natick, MA). Differences in M50 values were assessed by using a repeated measures analysis of variance
(ANOVA). Differences in protein levels were assessed using a one-way ANOVA. Tukey's honestly significant difference (HSD) was used as a post-hot test. Correlation analysis was carried out between protein levels and M50 values. Data analysis was performed using Statistica v12 (Tulsa, OK), and graphics were done in Origin v9 (Northamptom, MA).

\section{RESULTS}

\section{Validation of siRNA Effects on NMDA Receptor Subunit Protein Levels}

Figure 1 shows VM protein levels of NMDAR subunit GluN1 and GluN2A from total homogenates of rats injected (intraVM) with, GluN1, GluN2A, GluN2A + GluN2D, or control (scramble) siRNA. Microinjections of siRNA against GluN1 produced a significant $41 \%(\mathrm{SEM}=3.22)$ decrease in the expression of NMDAR GluN1 (Figure 1a) when compared against control $\left(t_{(20)}=5.38 ; p<0.0001\right)$. Similarly, a significant $39 \%(\mathrm{SEM}=5.26)$ reduction of NMDAR GluN2A expression was observed in rats injected with siRNA against GluN2A (Figure 1b) $\left(t_{(21)}=4.48 ; p<0.0001\right)$. For rats injected with GluN2A and GluN2D siRNA combination, a significant $42 \%(\mathrm{SEM}=4.67)$ decrease of NMDAR GluN2A expression (Figure 1c) $\left(t_{(14)}=3.78 ; p<0.001\right)$, but no specific signal was detected for the GluN2D expression in any of the present experimental conditions (control or in the presence of the Glu2D siRNA). Although there is evidence that GluN2D protein might be expressed at low levels in the VM (Dunah et al, 1996), similar difficulties had been experienced by others (Schilström et al, 2006). This is not related to the lack of protein detection by the antibody used, because a strong GluN2D immunosignal was detected in the hippocampus and thalamus brain areas enriched of this NMDAR subunit (Supplementary Figure 2). To test the selectivity of the siRNA for GluN2A, GluN2D, or their combination we tested the expression of GluN2B NMDAR in a subset of subjects. The results show no effect $\left(\mathrm{F}_{(3,12)}=0.315 ; p>0.05\right)$ on the expression of GluN2B receptor (Supplementary Figure 3).

\section{VM Downregulation of the GluN1 subunit, but not GluN2A, Strongly Reduced Electrically Evoked Reward}

Figure 2 shows for representative subjects the behavioral effects observed 24-h after the last siRNA injection for the different sequences used. Intra-VM injections of the inactive RNA sequence did not produce significant changes of the curve that relates the nose-poke rate as function of pulse frequency (Figure 2a). In contrast, intra-VM injections of the siRNA for GluN1 produced a rightward displacement of this curve. This displacement implies that the manipulation produced a reduction of the rewarding effectiveness of the stimulation (Figure 2b). Figure 2c-e shows that intra-VM injection of GluN2A, GluN2D alone, or their combination, did not produce any significant changes in the rate $v s$ pulsefrequency curves. Intra-VM injections of PPPA produced, regardless of the siRNA manipulation, a leftward displacement of the curve that relates the nose-poke rate as a function of pulse frequency, so less stimulation was necessary to obtain a given level of performance (Figure 2a-e). 


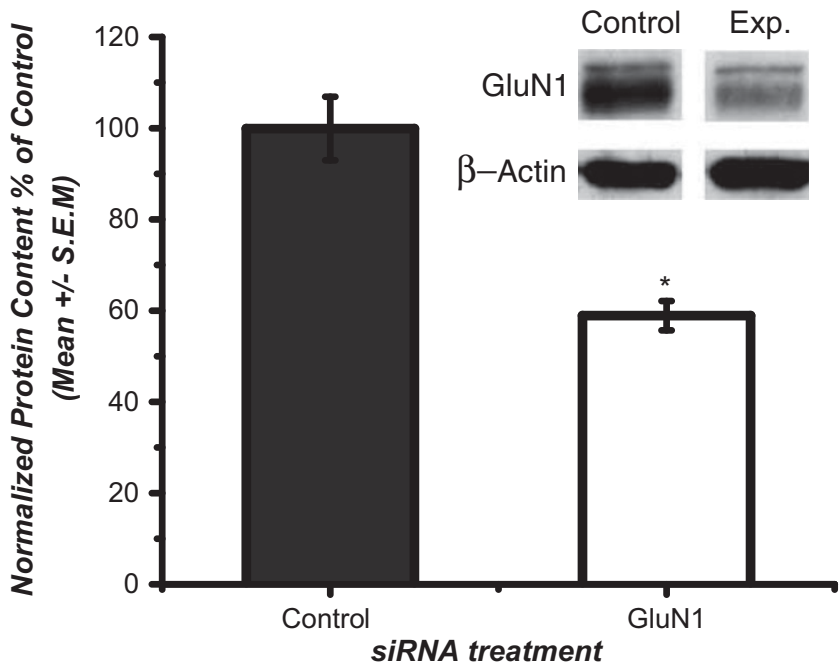

b

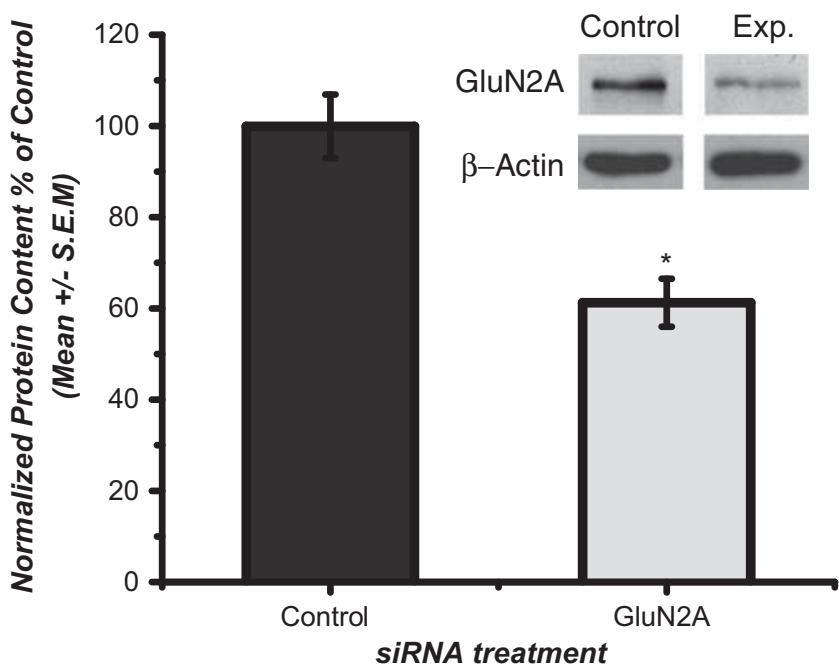

C

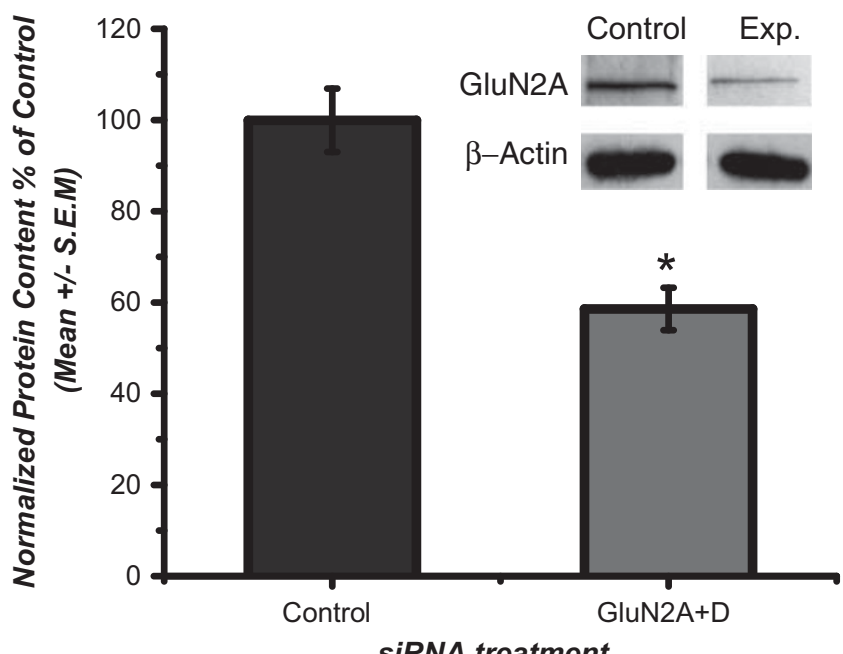

Figure 3 shows the average changes in M50 values (Figure 3a) and in maximal response (Figure 3b), expressed as $\%$ of baseline, for all the subjects that were injected with the inactive (control) and active siRNA. Analysis of variance confirms a significant effect of treatment $\left(\mathrm{F}_{(4,45)}=8.45\right.$ $p<0.0001)$. Post-hoc test shows that intra-VM injection of GluN1 produced a significant $25.5 \%(\mathrm{SEM}=3.67)$ increase in M50 values when compared against the rats that were injected with the non-active RNA. Although the maximum response rate (Figure $3 \mathrm{~b}$ ) was lower after rats received siRNA microinjections against GluN2A, GluN2D, and GluN2A $+2 \mathrm{D}$, this reduction was not statistically significant $\left(\mathrm{F}_{(4,45)}=1.26 ; p=0.299\right)$.

Figure 4 shows the correlation between the levels of increase in M50 observed in those rats which GluN1 receptor was reduced and the amount of protein downregulation measured in the VM. This result indicates a positive significant correlation between the maximum percentage change in reward threshold and the percentage change in protein reduction $(r=0.652 ; p<0.05)$. The increase in the reward threshold was directly related to the level of downregulation in GluN1 receptor; the reduction in GluN1 expression explains 36\% of the increase in reward threshold.

\section{Pharmacological GluN2A-Containing NMDAR Blockade Enhanced Brain Stimulation Reward Regardless of the siRNA Treatment}

We tested if reduction of the expression of the different NMDARs would impact intra-VM injections of PPPA. Despite the fact that PPPA displays only a modest pharmacological selectivity for GluN2A- vs GluN2B-containing NMDARs, we have previously shown that enhancement of electrically evoked reward following a wide range of intra-VM PPPA infusion is associated with GluN2Acontaining NMDA (Bergeron and Rompré, 2013; Ducrot et al, 2013). As expected, PPPA enhanced the rewarding effectiveness of the electrical stimulation and produced an increase in the maximum response rate. The magnitude of the leftward and upward displacement of the rate $v s$ pulsefrequency function can be appreciated in Figure 2. The maximum displacement of the M50 was obtained in average $19 \min (\mathrm{SEM}=1.76)$ after the PPPA injection, whereas the

Figure I siRNA microinjections against NMDARs produce a decrease in GluNI and Glun2A subunit immunoreactivity in the VM. (a) The inset of the figure shows samples of reactivity obtained for $10 \mu \mathrm{g}$ of $\mathrm{VM}$ homogenate from those rats treated with the control scramble siRNA, or against GluNI. The bar histogram shows the normalized levels of NMDA GluNI receptor against control. (b) The inset of the figure shows samples of reactivity obtained for $20 \mu \mathrm{g}$ of $\mathrm{VM}$ homogenate from those rats treated with the control scramble siRNA, or against GluN2A. The bar histogram shows the normalized levels of NMDA GluN2A receptor against control. (c) The inset shows samples of reactivity obtained for $20 \mu \mathrm{g}$ of VM homogenate from those rats treated with the control scramble siRNA, or against GluN2A and GluN2D. Only Glun2A was detected in our experiment. The bar histogram shows the normalized levels of NMDA GluN2A receptor against control. The levels of GluNI and GluN2A were detected using specific antibodies and normalized to $\beta$-actin. Asterisks indicate significant differences (* $p<0.001$; Student's t-test). 
a

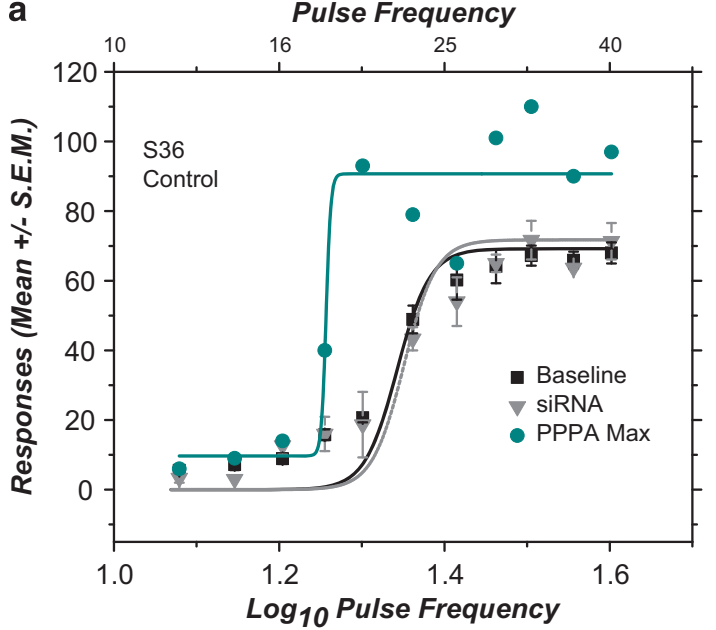

C

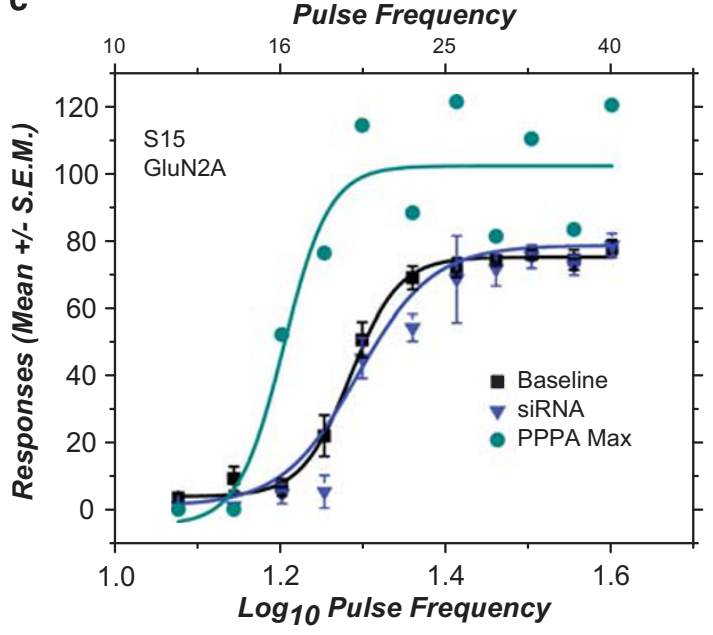

e

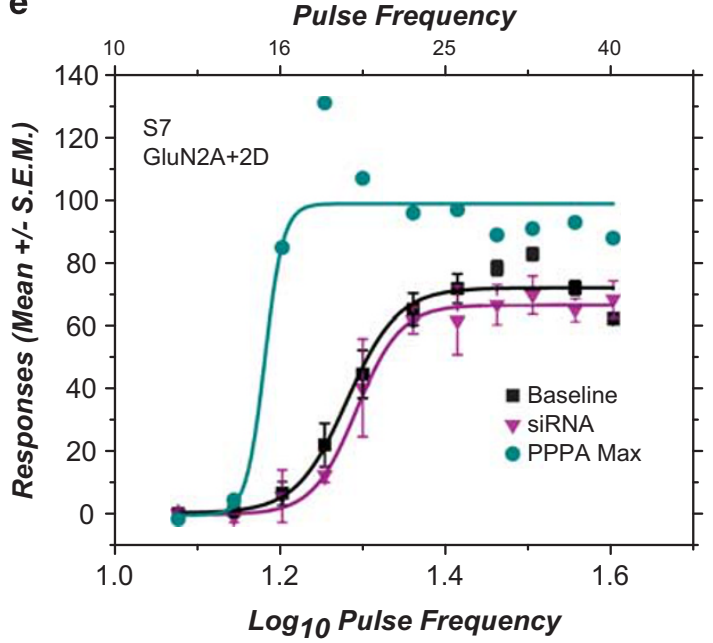

b

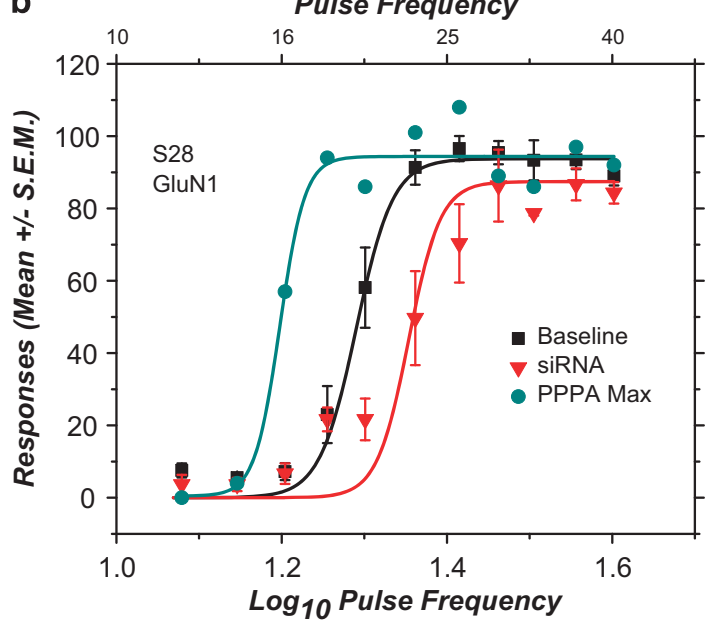

d

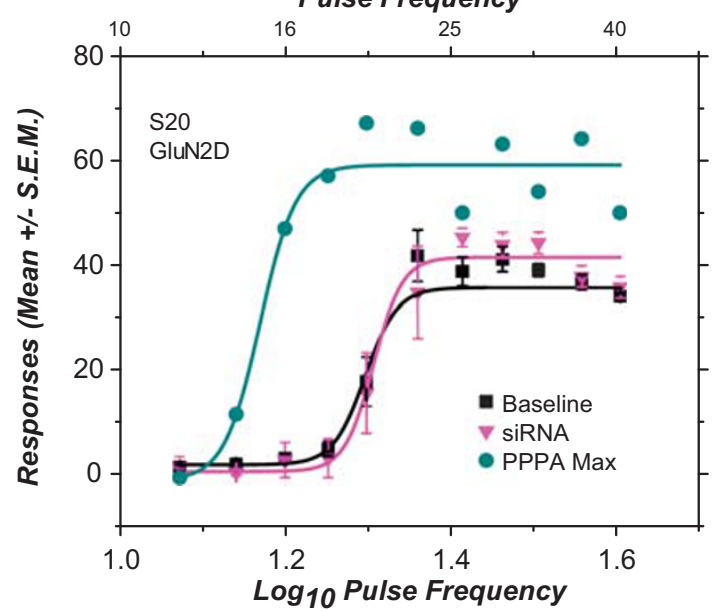

Figure 2 Rate-frequency curves for selected subjects show the effect of the different siRNA treatment and intra-VM injection of PPPA (0.825 nmol per $0.5 \mu \mathrm{l}$ per side). (a) Treatment with scramble siRNA ( $0.5 \mu \mathrm{g}$ per side) produced no effect on the curve that relates the nose-poke rate with the electrical pulse trains. The baseline curve and the one obtained 24-h after the last siRNA injection of overlap. Intra-VM injection of PPPA produced a leftward and upward shift of rate-frequency curve. (b) Treatment with siRNA (0.5 $\mu \mathrm{g}$ per side) against GluNI produced a decrease of the rewarding effects that arise from the electrical stimulation; the red curve obtained 24-h after the last siRNA injection is displaced to the right of the baseline curve that is shown in black. The siRNA treatment did not alter PPPA's effectiveness to produce an enhancement in reward. (c) Treatment with siRNA (0.5 $\mu \mathrm{g}$ per side) against GluN2A was ineffective in altering the rewarding effects that arise from the electrical stimulation; the blue curve obtained 24-h after the last siRNA injection overlap with that obtained during baseline. Intra-VM injection of PPPA produced a leftward and upward shift of rate-frequency curve. (d) Treatment with siRNA $(0.5 \mu \mathrm{g}$ per side) against GluN2D and (e) the combination of GluN2A and GluN2D were ineffective in altering the rewarding effects that arise from the electrical stimulation, respectively, the magenta and dark magenta curves overlap with those obtained during baseline. siRNA treatment did not alter the effectiveness of intra-VM injection of PPPA, this drug produced a leftward and upward shift of rate-frequency curves. 
a
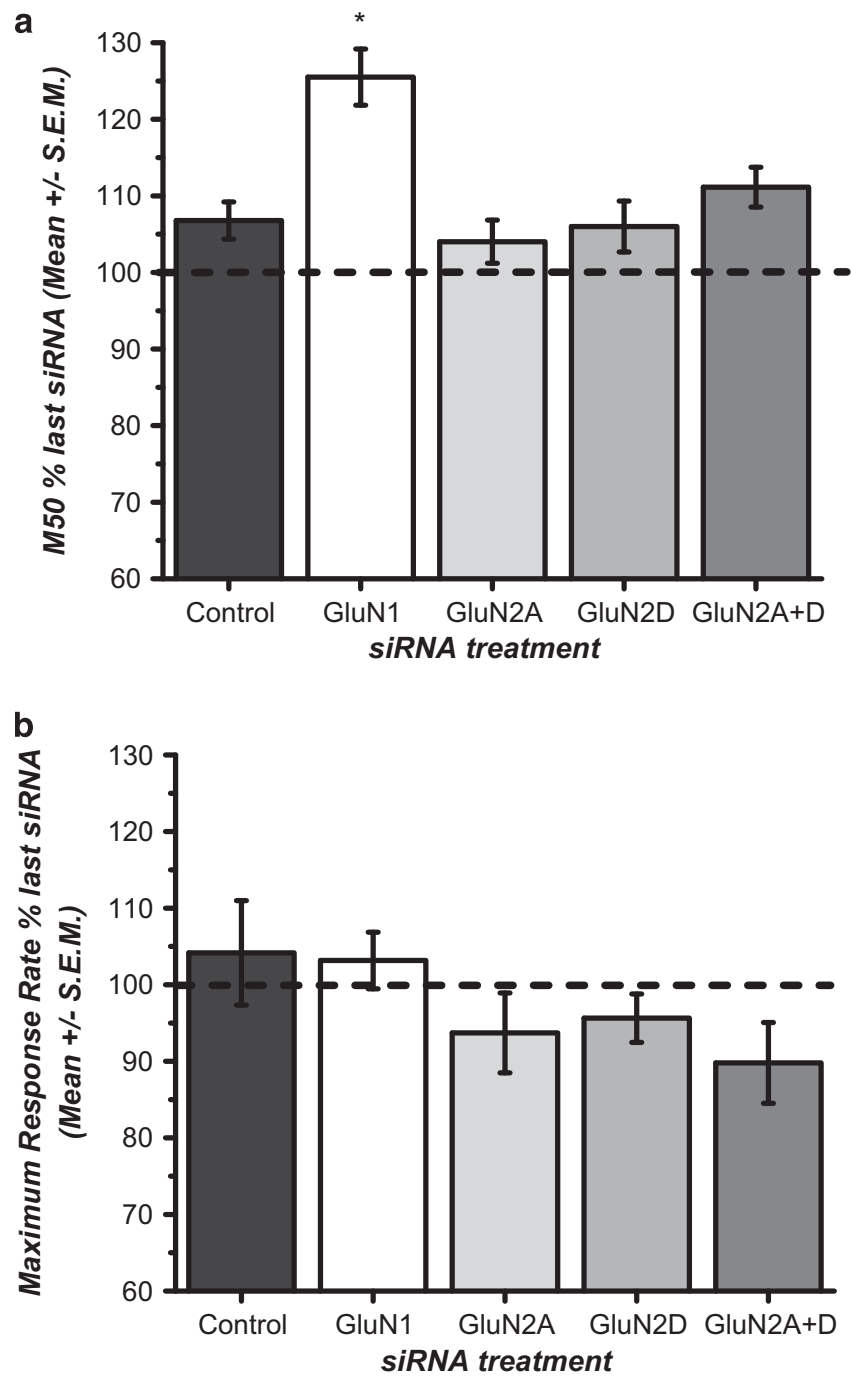

Figure 3 Bar graphs representing the averages of maximum change in stimulation threshold and maximum response rate for all siRNA treatments. (a) Treatment with siRNA against GluNI receptor produce a significant increase in the stimulation threshold $\left(F_{(4.45)}=8.45 ; p<0.000 \mathrm{I}\right)$. (b) None of the siRNA treatments produced a significant change in maximum response rate $\left(F_{(4,45)}=1.26 ; p=0.299\right)$.

change in maximum rate was obtained after $25 \mathrm{~min}$ (SEM $=$ 3.13). There was no significant difference between the groups in their timing for M50 displacement $\left(\mathrm{F}_{(4,43)}=1.58\right.$; $p>0.05)$ or maximum response rate displacement $\left(\mathrm{F}_{(4,43)}=0.97 ; p>0.05\right)$. Figure 5 shows the effect of PPPA for each of the treatment groups on the maximum decrease of M50 (Figure 5a) and the maximum increase in response rate (Figure 5b). PPPA produced an average 25.5\% $(\mathrm{SEM}=1.37)$ reduction in M50 value. The ANOVA yielded a significant effect of treatment $\left(\mathrm{F}_{(5,44)}=14.88, p<0.0001\right)$ and post-hoc test showed that the M50 values, when compared with subjects that received saline, were significantly reduced regardless of the siRNA treatment. PPPA also produced comparable increases in the maximum response rate regardless of the siRNA treatment group, an average of $42 \%(S E M=5.12)$. The ANOVA yielded a significant effect of treatment $\left(\mathrm{F}_{(5,44)}=2.58, p<0.039\right)$ and

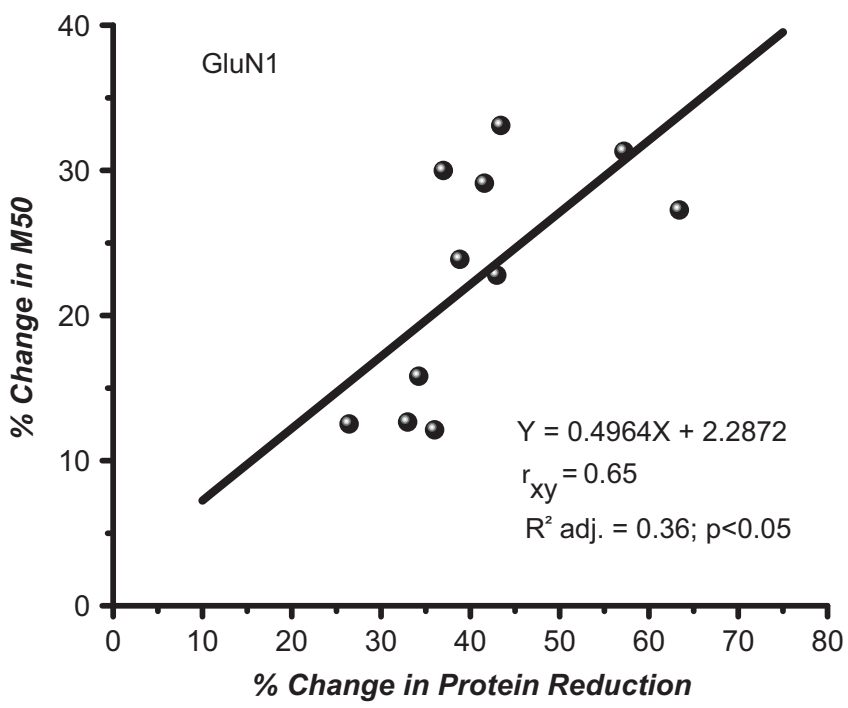

Figure 4 Scatterplot showing the positive relation between the change in stimulation threshold and the change in protein reduction for those rats that were injected with siRNA against GluNI. There is a positive correlation $\left(r_{(x, y)}=0.652 ; p<0.05\right)$ between the reduction in GluNI and the amount of change in M50, so that the greatest the protein reduction the biggest was the observed increase in stimulation threshold. The line represents the fit of the linear equation shown in the graphs and the $R^{2}$ adj shows the proportion of explained variance in $M 50$ by the changes in GluNI expression.

post-hoc test showed that maximum response rate, when compared with subjects who received saline, was significantly increased regardless of the siRNA treatment.

\section{DISCUSSION}

The main finding of the present study is that downregulation of VM NMDAR following repeated microinjections of siRNA targeting the GluN1 subunit produces an attenuation of the reward signal induced by DR electrical stimulation. This attenuation is inferred by a rightward displacement of the rate $v s$ pulse-frequency curve and the absence of a significant change in the maximum response rate, a measure that has been traditionally associated with changes in performance capacity (Miliaressis et al, 1986). This attenuation effect is most likely because of a decrease in the glutamatergic excitability onto DA neurons. Rewarding electrical stimulation produces an increase in DA cell firing and DA release (Hernández and Shizgal, 2009; Moisan and Rompre, 1998) and a large body of evidence shows that reward induced by brain electrical stimulation is highly sensitive to changes in VM DA neurotransmission. Drugs that enhance synaptic DA levels such as DA transporter blocker GBR12909 produces a leftward displacement of the curve that relates response rate to stimulation frequency (Rompré and Bauco, 1990). Opposite effects are obtained with DA receptor antagonists such as haloperidol and raclopride (Nakajima and Patterson, 1997). In further support for this hypothesis are the results showing that glutamate-containing terminals establish synaptic contacts with DA neurons (Carr and Sesack, 2000; 
a
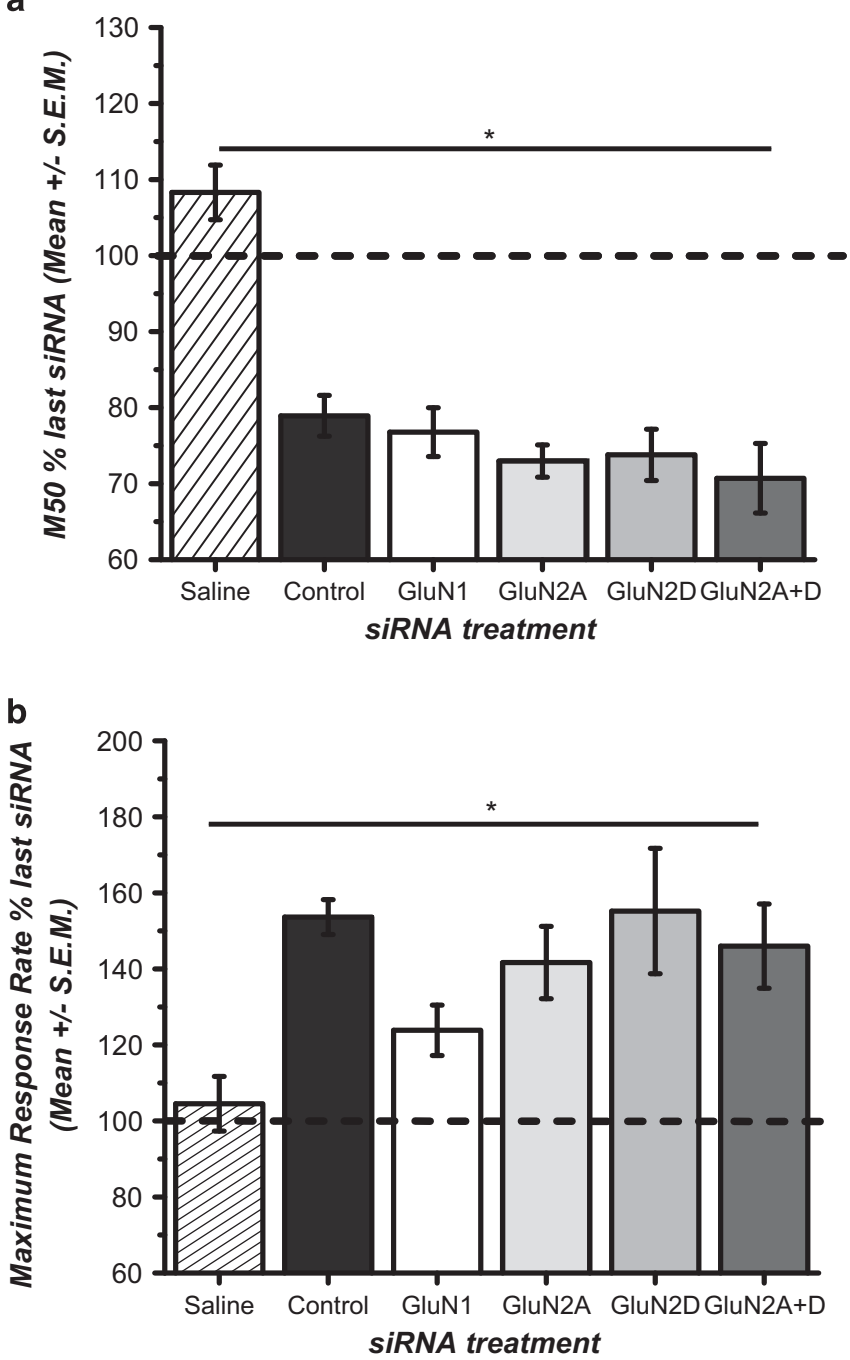

Figure 5 Bar graphs representing the Averages of maximum change in stimulation threshold and maximum response rate after intra-VM injection of saline or PPPA (0.82 pmol per $0.5 \mu \mathrm{l}$ per side) for all subject that received the different siRNA treatments. (a) PPPA injection, when contrasted against those subjects that received saline produced a significant reduction in self-stimulation threshold. This reduction was similar regardless of the NMDAR(s) targeted by the siRNA treatment $\left(F_{(5,44)}=\mid 4.88\right.$, $p<0.000 \mathrm{I}$ ). (b) Similarly, PPPA when contrasted against those subjects that received saline produced a significant increase in maximum response rate, and such increase was indifferent to the siRNA treatment $\left(F_{(5,44)}=2.58\right.$, $p<0.039)$.

Omelchenko and Sesack, 2009) and that rewarding electrical stimulation is associated with an increase in VM glutamate release (You et al, 2001).

Both serotonergic and non-serotonergic DR neurons project to the $\mathrm{VM}$ and a recent study has shown that selective activation of DR non-serotonergic cell bodies, or their VM terminals, sustains operant responding and induces place preference (Mcdevitt et al, 2014). McDavitt et al, (2014) also showed that stimulation of this DR to VM pathway generates glutamatergic excitatory postsynaptic current in VM dopamine neurons, and that the rewarding effect associated with its activation is blocked by a selective dopamine D1 antagonist. A substantial number of DR neurons express VGluT3, which constitutes a major part of the DR efferent projection to the ventral midbrain (Hioki et al, 2010; Qi et al, 2014; Watabe-Uchida et al, 2012). Dorsal raphe glutamatergic neurons also establish asymetrical synaptic connections with VM dopamine neurons (Qi et al, 2014). Altogether, these findings strongly suggest that reward can be induced by activation of DR glutamatergic afferent inputs to VM DA neurons. Consistently, two recent optogenetic studies have shown that selective activation of DR glutamatergic releasing neurons or glutamatergic VM terminals is rewarding (Liu et al, 2014; Qi et al, 2014).

The hypothesis that the rewarding signal is transmitted to the VM by glutamate raises the possibility that the enhanced glutamate release produced by repeated electrical stimulations alter the expression of the NMDARs. It was shown for instance that repeated treatments with drugs of abuse known to enhance brain stimulation reward alter the expression of GluN2A and GluN2B, and their respective mRNA, in different limbic brain regions (Barr et al, 2014; Gipson and Reissner, 2013; Kindlundh-Högberg et al, 2008). Carlezon et al, (2001) compared the expression of GluN1 in the ventral tegmental area between rats that self-stimulated for one hour consecutively and rats that received no stimulation and found no difference between the two groups. This result suggests that repeated rewarding stimulations do not alter VTA NMDARs expression.

NMDARs are heterodimers composed of two obligatory GluN1 subunits with GluN2 and/or GluN3 subunits. Since GluN1 subunits are common to all NMDARs, the siRNA directed toward GluN1 should have produced a nonselective downregulation of NMDAR expression. Because other siRNA manipulations produced a significant reduction of GluN2A without any reliable change in self-stimulation behavior, we are forced to conclude that the NMDARs mediating the reward signal are not composed of GluN2A subunits. They are also unlikely composed of GluN2B, since no reward enhancement was observed in a previous pharmacological study with a selective GluN2B antagonist injected into the VM (Bergeron and Rompré, 2013). One hypothesis then is that the relevant NMDARs are heterodimers composed of GluN1 and GluN3 subunits. In support to this possibility, Yuan et al, (2013) recently identified GluN3A mediated current in VM DA neurons and showed that GluN3A-containing NMDARs mediate DA neurons plasticity induced by cocaine reward. Alternative hypotheses are that the reduction in GluN2A receptor was not sufficient to modify the excitatory drive of glutamate or that the behavioral change was not reliably detectable by the curve-shift paradigm and some other more sensitive paradigm, like the reward-mountain paradigm (Hernandez et al, 2010) would be necessary to detect such changes. The likelihood of these hypotheses, however, is very low given that a comparable downregulation in the GluN1 receptor produced a significant reduction in reward pursuit that was readily detectable by the behavioral paradigm that we used.

In accordance with previous results, ventral midbrain microinjection of the NMDA antagonist, PPPA, produced a leftward and upward shift of the pulse-frequency curve reflecting an enhancement of reward and performance (see Bergeron and Rompré, 2013; Ducrot et al, 2013). This shows that VM glutamate also exerts a negative modulation on 
reward. The main idea to account for the opposite effects of glutamate is that they are mediated by different subtypes of NMDARs localized on different afferent inputs to VM DA neurons or on DA cells themselves (see Supplementary Figure 4). If inhibitory interneurons that maintained a strong inhibitory drive over DA neurons express GluN2A, blockade of these receptors would then allow DA neurons to pass from a silent state into a tonic firing state; this state will increase the probability that a glutamate signal initiates burst firing. Glutamate participates in this form of feedforward inhibition, altering the plasticity of local inhibitory neurons and dopamine cell firing as in-vitro electrophysiological recordings had shown (Bonci and Malenka, 1999; Nugent and Kauer, 2008). But how can we explain the fact that VM microinjection of PPPA, a preferred GluN2A antagonist, still enhanced reward in animals that had a reduction in NMDARs, and particularly in GluN2A subunits? A tantalizing explanation is that downregulation of NMDARs was not sufficient to alter the rewardenhancing effect of the drug. Again, this is rather unlikely as the reduction in GluN1 was sufficient to reduce reward but not the reward-enhancing effect of PPPA. The cellular machinery required for silencing the targeted RNA is primarily located in the nucleus of the cell. Unlike pharmacological manipulations, local brain infusion of siRNA only downregulates receptors of those cells that have their cell bodies located in the VM; it is ineffective at reducing receptors located on afferent terminals. Inhibitory interneurons establish connection with dendritic shafts which render their physiological capacity to be a stronger inhibitory input but make them very important in coordinating neighboring cell activity. This characteristic contrasts with that of inhibitory afferents which establish somatic connections and therefore believed to establish a stronger inhibition (Omelchenko and Sesack, 2009). Thus, our siRNA manipulation might have altered the neural coordination within the VM, but did not dramatically alter the inhibition of DA neurons. If the main inhibitory source comes from VM afferents then PPPA blockade of GluN2A occurs on presynaptic receptors. There is evidence that presynaptic NMDARs are localized in GABAergic terminals of several brain areas including the VM (Paquet and Smith, 2000) and their role seems to modulate the strength of synaptic transmission by varying the likelihood of neurotransmitter release (Pinheiro and Mulle, 2008). In most brain areas, the presence of presynaptic NMDARs contribute to release of inhibitory neurotransmitter in the cerebral cortex (Mathew and Hablitz, 2011; Ren et al, 2007), lateral amygdala (Humeau et al, 2003), cerebellum (Bidoret et al, 2009), entorhinal cortex (Chamberlain et al, 2008), and visual cortex ( $\mathrm{Li}$ et al, 2008). In general these studies demonstrate that inhibition can be potentiated by axoaxonic contacts without the need for somatic interneuron firing; such mechanism alters the weight between excitatory and inhibitory inputs and therefore alters the output of the neuronal circuit. The idea of a presynaptic control of GABA release is also supported by our PPPA results where pharmacological blockade of GluN2A NMDARs produced a similar enhancement in reward as the one observed in the control subjects and in previous studies (Bergeron and Rompré, 2013; Ducrot et al, 2013), whereas intra-VM downregulation of its mRNA remained without effect.
The rapid effects of ketamine on mood deficit at a dose that acts primarily as NMDAR antagonist (Berman et al, 2000) supports the importance of glutamate and its regulation on mood disorders. Although the clinical effect of ketamine is mainly attributed to blockade of GluN2B NMDAR (Fond et al, 2014; McGirr et al, 2014), our results show that GluN2A-NMDARs located on afferent terminals of the VM are the most likely source of inhibition that modulates reward seeking, suggesting a complex circuitry that was not fully appreciated in prior pharmacological studies. Our results suggest that a dysregulation or an enhancement of VM GluN2A-mediated neurotransmission can lead to severe alteration of mood characterized by anhedonia. It would be important to identify with the use of new technologies, like optogenetics and designer receptors exclusively activated by a designer drug (DREADD), that offers temporal and regional specific control over targeted neural cell types and receptors which GABAergic afferents to the VM regulate brain reward function. One likely candidate is the ventral pallidum which sends dense GABAergic connections to the VM (Kalivas et al, 1993), regulates DA neurons firing (Floresco et al, 2003) and affects rewardrelevant tasks (see (Smith et al, 2009) for a rewiew). These strategies may provide important insights into the neural circuitry underlying reward pursuit and would shed light on disorders in which the reward signal is not adequately integrated or weighted as it the case in depression.

\section{FUNDING AND DISCLOSURE}

The authors declare no conflict of interest.

\section{ACKNOWLEDGEMENTS}

The present research was supported by NSERC grant \#119057 to Pierre-Paul Rompré, NSERC fellowship to Giovanni Hernandez. We thank Claude Bouchard for is excellence assistance and Sandra M Boye for her helpful suggestions.

\section{REFERENCES}

Barr JL, Forster GL, Unterwald EM (2014). Repeated cocaine enhances ventral hippocampal-stimulated dopamine efflux in the nucleus accumbens and alters ventral hippocampal NMDA receptor subunit expression. J Neurochem 130: 583-590.

Bergeron S, Rompré P-P (2013). Blockade of ventral midbrain NMDA receptors enhances brain stimulation reward: a preferential role for GluN2A subunits. Eur Neuropsychopharmacol 23: 1623-1635.

Berman RM, Cappiello A, Anand A, Oren DA, Heninger GR, Charney DS et al (2000). Antidepressant effects of ketamine in depressed patients. Biol Psychiatry 3223 47: 351-354.

Bidoret C, Ayon A, Barbour B, Casado M (2009). Presynaptic NR2A-containing NMDA receptors implement a high-pass filter synaptic plasticity rule. Proc Natl Acad Sci USA 106: 14126-14131.

Bonci A, Malenka RC (1999). Properties and plasticity of excitatory synapses on dopaminergic and GABAergic cells in the ventral tegmental area. J Neurosci 19: 3723-3730.

Carlezon W, Todtenkopf M, McPhie D, Pimentel P, Pliakas A, Stellar JR et al (2001). Repeated exposure to rewarding brain stimulation downregulates GluR1 expression in the ventral tegmental area. Neuropsychopharmacology 25: 234-241. 
Carr DB, Sesack SR (2000). Projections from the rat prefrontal cortex to the ventral tegmental area: target specificity in the synaptic associations with mesoaccumbens and mesocortical neurons. J Neurosci 20: 3864-3873.

Chamberlain SEL, Yang J, Jones RSG (2008). The role of NMDA receptor subtypes in short-term plasticity in the rat entorhinal cortex. Neural Plast 2008: 872456.

Charlety PJ, Grenhoff J, Chergui K, De la Chapelle B, Buda M, Svensson TH et al (1991). Burst firing of mesencephalic dopamine neurons is inhibited by somatodendritic application of kynurenate. Acta Physiol Scand 142: 105-112.

Chergui K, Charléty PJ, Akaoka H, Saunier CF, Brunet JL, Buda M et al (1993). Tonic activation of NMDA receptors causes spontaneous burst discharge of rat midbrain dopamine neurons in vivo. Eur J Neurosci 5: 137-144.

Cornish JL, Nakamura M, Kalivas PW (2001). Dopamineindependent locomotion following blockade of N-methyl-Daspartate receptors in the ventral tegmental area. J Pharmacol Exp Ther 298: 226-233.

David V, Durkin TP, Cazala P (1998). Rewarding effects elicited by the microinjection of either AMPA or NMDA glutamatergic antagonists into the ventral tegmental area revealed by an intracranial self-administration paradigm in mice. Eur J Neurosci 10: $1394-1402$.

Dobi A, Margolis EB, Wang H-L, Harvey BK, Morales M (2010). Glutamatergic and nonglutamatergic neurons of the ventral tegmental area establish local synaptic contacts with dopaminergic and nondopaminergic neurons. J Neurosci 30: 218-229.

Ducrot C, Fortier E, Bouchard C, Rompré P-P (2013). Opposite modulation of brain stimulation reward by NMDA and AMPA receptors in the ventral tegmental area. Front Syst Neurosci 7: 57.

Dunah AW, Yasuda RP, Wang YH, Luo J, Dávila-García M, Gbadegesin $\mathrm{M}$ et al (1996). Regional and ontogenic expression of the NMDA receptor subunit NR2D protein in rat brain using a subunit-specific antibody. J Neurochem 67: 2335-2345.

Floresco SB, West AR, Ash B, Moore H, Grace AA (2003). Afferent modulation of dopamine neuron firing differentially regulates tonic and phasic dopamine transmission. Nat Neurosci 6: 968-973.

Fond G, Loundou A, Rabu C, Macgregor A, Lançon C, Brittner M et al (2014). Ketamine administration in depressive disorders: a systematic review and meta-analysis. Psychopharmacology (Berl) 231: 3663-3676.

French ED, Mura A, Wang T (1993). MK-801, phencyclidine (PCP), and PCP-like drugs increase burst firing in rat A10 dopamine neurons: comparison to competitive NMDA antagonists. Synapse 13: 108-116.

Geisler S, Derst C, Veh RW, Zahm DS (2007). Glutamatergic afferents of the ventral tegmental area in the rat. J Neurosci 27: 5730-5743.

Gipson C, Reissner K (2013). Reinstatement of nicotine seeking is mediated by glutamatergic plasticity. Proc Natl Acad Sci USA 110: $10-15$.

Grace AA, Bunney BS (1984). The control of firing pattern in nigral dopamine neurons: burst firing. J Neurosci 4: 2877-2890.

Greenberg P, Corey-Lisle PK, Birnbaum H, Marynchenko M, Claxton A (2004). Economic implications of treatment-resistant depression among employees. Pharmacoeconomics 22: 363-373.

Hernandez G, Breton Y-A, Conover K, Shizgal P (2010). At what stage of neural processing does cocaine act to boost pursuit of rewards? PLoS One 5: e15081.

Hernández G, Shizgal P (2009). Dynamic changes in dopamine tone during self-stimulation of the ventral tegmental area in rats. Behav Brain Res 198: 91-97.

Hioki H, Nakamura H, Ma Y-F, Konno M, Hayakawa T, Nakamura KC et al (2010). Vesicular glutamate transporter 3-expressing nonserotonergic projection neurons constitute a subregion in the rat midbrain raphe nuclei. J Comp Neurol 518: 668-686.
Humeau Y, Shaban H, Bissière S, Lüthi A (2003). Presynaptic induction of heterosynaptic associative plasticity in the mammalian brain. Nature 426: 841-845.

Kalivas PW, Churchill L, Klitenick MA (1993). GABA and enkephalin projection from the nucleus accumbens and ventral pallidum to the ventral tegmental area. Neuroscience 57: 1047-1060.

Karreman M, Westerink BH, Moghaddam B (1996). Excitatory amino acid receptors in the ventral tegmental area regulate dopamine release in the ventral striatum. J Neurochem 67: 601-607.

Kindlundh-Högberg AMS, Blomqvist A, Malki R, Schiöth HB (2008). Extensive neuroadaptive changes in cortical genetranscript expressions of the glutamate system in response to repeated intermittent MDMA administration in adolescent rats. BMC Neurosci 9: 39.

Kretschmer BD (1999). Modulation of the mesolimbic dopamine system by glutamate: role of NMDA receptors. J Neurochem 73: 839-848.

Kupfer DJ, Ellen F, Perel JM (1989). The advantage of early treatment intervention in recurrent depression. Arch Gen Psychiatry 46: 771-775.

Li YH, Han TZ, Meng K (2008). Tonic facilitation of glutamate release by glycine binding sites on presynaptic NR2B-containing NMDA autoreceptors in the rat visual cortex. Neurosci Lett 432: 212-216.

Liu Z, Zhou J, Li Y, Hu F, Lu Y, Ma M et al (2014). Dorsal raphe neurons signal reward through 5-HT and glutamate. Neuron 81: $1360-1374$.

Maeng S, Zarate CA, Du J, Schloesser RJ, McCammon J, Chen G et al (2008). Cellular mechanisms underlying the antidepressant effects of ketamine: role of alpha-amino-3-hydroxy-5-methylisoxazole-4-propionic acid receptors. Biol Psychiatry 63: 349-352.

Mathew SS, Hablitz JJ (2011). Presynaptic NMDA receptors mediate IPSC potentiation at GABaergic synapses in developing rat neocortex. PLoS One 6: e17311.

Mcdevitt RA, Tiran-cappello A, Harvey BK, Bonci A, Shen H, Balderas I et al (2014). Serotonergic versus nonserotonergic dorsal raphe projection neurons: differential participation in reward article serotonergic versus nonserotonergic dorsal raphe projection neurons: differential participation in reward circuitry. Cell Rep 8: 1857-1869.

McGirr A, Berlim MT, Bond DJ, Fleck MP, Yatham LN, Lam RW (2014). A systematic review and meta-analysis of randomized, double-blind, placebo-controlled trials of ketamine in the rapid treatment of major depressive episodes. Psychol Med 10: 1-12.

Miliaressis E, Rompre PP, Laviolette P, Philippe L, Coulombe D (1986). The curve-shift paradigm in self-stimulation. Physiol Behav 37: 85-91.

Moisan J, Rompre PP (1998). Electrophysiological evidence that a subset of midbrain dopamine neurons integrate the reward signal induced by electrical stimulation of the posterior mesencephalon. Brain Res 786: 143-152.

Nakajima S, Patterson RL (1997). The involvement of dopamine D2 receptors, but not D3 or D4 receptors, in the rewarding effect of brain stimulation in the rat. Brain Res 760: 74-79.

Nestler EJ, Carlezon WA (2006). The mesolimbic dopamine reward circuit in depression. Biol Psychiatry 59: 1151-1159.

Nugent FS, Kauer JA (2008). LTP of GABAergic synapses in the ventral tegmental area and beyond. J Physiol 586: 1487-1493.

Omelchenko N, Sesack SR (2009). Ultrastructural analysis of local collaterals of rat ventral tegmental area neurons: GABA phenotype and synapses onto dopamine and GABA cells. Synapse 63: 895-906.

Overton PG, Clark D (1997). Burst firing in midbrain dopaminergic neurons. Brain Res Brain Res Rev 25: 312-334.

Paquet M, Smith Y (2000). Immunoreactivity in GABAergic terminals in rat brain. J Comp Neurol 347: 330-347. 
Paxinos G, Watson C (2007). The Rat Brain in Stereotaxic Coordinates. Sixth Edition. Elsevier Acad Press, 170: 547-612.

Pinheiro PS, Mulle C (2008). Presynaptic glutamate receptors: physiological functions and mechanisms of action. Nat Rev Neurosci 9: 423-436.

Qi J, Zhang S, Wang H-L, Wang H, de Jesus Aceves Buendia J, Hoffman AF et al (2014). A glutamatergic reward input from the dorsal raphe to ventral tegmental area dopamine neurons. Nat Commun 5: 5390.

Ren M, Yoshimura Y, Takada N, Horibe S, Komatsu Y (2007). Specialized inhibitory synaptic actions between nearby neocortical pyramidal neurons. Science 316: 758-761.

Rompré PP, Bauco P (1990). GBR 12909 reverses the SCH 23390 inhibition of rewarding effects of brain stimulation. Eur $J$ Pharmacol 182: 181-184.

Salahpour A, Medvedev IO, Beaulieu J-M, Gainetdinov RR, Caron MG et al (2007). Local knockdown of genes in the brain using small interfering RNA: a phenotypic comparison with knockout animals. Biol Psychiatry 61: 65-69.

Schilström B, Yaka R, Argilli E, Suvarna N, Schumann J, Chen BT et al (2006). Cocaine enhances NMDA receptor-mediated currents in ventral tegmental area cells via dopamine D5 receptor-dependent redistribution of NMDA receptors. J Neurosci 26: 8549-8558.
Schultz W (2010). Dopamine signals for reward value and risk: basic and recent data. Behav Brain Funct 6: 24 .

Smith KS, Tindell AJ, Aldridge JW, Berridge KC (2009). Ventral pallidum roles in reward and motivation. Behav Brain Res 196: 155-167.

Strekalova T, Couch Y, Kholod N, Boyks M, Malin D, Leprince P et al (2011). Update in the methodology of the chronic stress paradigm: internal control matters. Behav Brain Funct 7: 9.

Watabe-Uchida M, Zhu L, Ogawa SK, Vamanrao A, Uchida N (2012). Whole-brain mapping of direct inputs to midbrain dopamine neurons. Neuron 74: 858-873.

You ZB, Chen YQ, Wise RA (2001). Dopamine and glutamate release in the nucleus accumbens and ventral tegmental area of rat following lateral hypothalamic self-stimulation. Neuroscience 107: 629-639.

Yuan T, Mameli M, O'Connor EC, Dey PN, Verpelli C, Sala C et al (2013). Expression of cocaine-evoked synaptic plasticity by GluN3A-containing NMDA receptors. Neuron 80: 1025-1038.

Zweifel LS, Parker JG, Lobb CJ, Rainwater A, Wall VZ, Fadok JP et al (2009). Disruption of NMDAR-dependent burst firing by dopamine neurons provides selective assessment of phasic dopamine-dependent behavior. Proc Natl Acad Sci USA 106: 7281-7288.

Supplementary Information accompanies the paper on the Neuropsychopharmacology website (http://www.nature.com/npp) 